\title{
Hydrodynamic scaling and wave force estimation of offshore structures
}

\author{
Jifu Zhou ${ }^{1,2} \cdot$ Ling Chen ${ }^{1,2} \cdot$ Xu Wang $^{1}$
}

Received: 9 June 2020 / Revised: 13 August 2020 / Accepted: 8 September 2020 / Published online: 28 October 2020

(C) The Chinese Society of Theoretical and Applied Mechanics and Springer-Verlag GmbH Germany, part of Springer Nature 2020

\begin{abstract}
Offshore structures are generally classified as small-scale structures or large-scale structures. Their wave forces are then estimated by Morison equation and diffraction/radiation theories, respectively. However, the classification criterion is not well quantified. In the present paper, a numerical wave flume is established to simulate the wave forces acting on a fixed and vertical surface-piercing circular cylinder under linear waves. By solving Navier-Stokes equation and Euler equation with free surface involved, respectively, the viscous force and inertia force are separated accurately. The variation of viscous force and inertia force with the cylinder diameter to wave length ratio is discussed in detail. The scale intervals for significant viscous and diffraction effects are given. The error caused by neglecting viscous and diffraction forces is quantitatively analyzed. Based on these analysis, the concept of medium-scale structure is proposed and the classification criteria for small-, mediumand large-scale structures are given. In the meantime, the estimation methods of wave forces for different scales of structures are suggested.
\end{abstract}

Keyword Wave force $\cdot$ Viscous effect $\cdot$ Diffraction effect $\cdot$ Medium-scale structure $\cdot$ Numerical wave flume

\section{Introduction}

How to accurately estimate wave loads of an offshore structure is of primary importance in offshore engineering design. The components of wave loads are very complex. They are significantly influenced by viscous, diffraction, radiation and nonlinear effects, etc. These effects have important impact on the accurate estimation of wave loads on offshore structures. In order to clarify the composition and mechanism of wave loads, hydrodynamic and structural parameters have to be considered carefully. A typical example of investigating wave loads on a structure is to consider a fixed and vertical surface-piercing circular cylinder in the flow field of a regular wave.

\section{Jifu Zhou}

zhoujf@imech.ac.cn

1 CAS Key Laboratory for Mechanics in Fluid Solid Coupling Systems, Institute of Mechanics, Chinese Academy of Sciences, Beijing 100190, China

2 School of Engineering Sciences, University of Chinese Academy of Sciences, Beijing 100049, China
A simple dimensional analysis shows that any timeinvariant force $F$ on a fixed body may be written as follows [1]

$\frac{F}{\rho g H D^{2}}=f\left(\frac{d}{L}, \frac{H}{L}, \frac{D}{L}, R e\right)$,

where $R e$ is a characteristic Reynolds number, $D$ the diameter of the cylinder, $L$ the wavelength, $H$ the wave height, $d$ the water depth under still water level, $\rho$ the density of water, $g$ the acceleration due to gravitation. The wave depth parameter $d / L$ and the wave steepness $H / L$ can determine the incident wave. The diffraction parameter $D / L$ reflects the effect of wave diffraction or scattering. The characteristic Reynolds number $R e$ represents the viscous effect, flow separation in particular.

As yet both a theoretical analysis of the problem of flow separation and a theoretical analysis of nonlinear wave diffraction are difficult. Therefore, there has been no theoretical expression for wave loads on offshore structures yet. According to the experimental studies of Morison et al. [2], the wave forces on a pile are divided into two parts. One is due to the drag and the other due to the acceleration or decel- 
eration of water. Hence, the force per unit length experienced by the cylinder can be expressed as

$$
F=\frac{1}{2} C_{d} \rho D|U| U+C_{m} \rho \frac{\pi D^{2}}{4} \frac{\mathrm{d} U}{\mathrm{~d} t},
$$

in which $U$ and $\mathrm{d} U / \mathrm{d} t$ represent the undisturbed velocity and the acceleration of water, respectively, $C_{d}$ and $C_{m}$ represent drag coefficient and inertia coefficient, respectively.

Equation (2) is widely used for estimating the wave loads on offshore structures. Nonetheless, it is only applicable to structures of small scale, such as slender piles [3]. For structures of large scale, such as spar type platforms [4], storage tanks [5] or other structures involving components of relatively large section and complex geometry [6], etc., it is necessary to use methods based on diffraction theory for wave load estimation. Diffraction theory analyzes the fluid motion around a fixed or moving body in waves by describing pressure distribution over the body surface, which satisfy all the boundary conditions. The forces and moments are then calculated by integrating the pressure distribution over the wet surface of the body. An analytic solution to the linear wave diffraction problem is available for an isolated vertical circular cylinder extending from the seabed and piercing the free surface. This was treated by MacCamy and Fuchs [7] for general water depths.

Now, it seems simple to estimate wave loads of offshore structures provided whether the structures are of small or large scales is determined. For large structures, inertia force dominates and diffraction should be considered based on diffraction theory to calculate the wave forces. For small structures, drag force due to fluid viscosity dominates and the Morison formula with the coefficients elaborately chosen is usually used to calculate the wave forces. Here, the small structure implies that it is small enough in relation to the wavelength so that there is no wave scattering and uniform ambient acceleration can be assumed. Therefore, the diffraction parameter $D / L$ (i.e., the ratio of structure diameter $D$ to wavelength $L$ ) is usually used to define structure scale. Hogben [8] recommended $D / L<0.2$ as a criterion for small structures based on data in Chakrabarti and Tam [9] and on consideration of the range of wavelength over which it is reasonable to assume uniform acceleration by reference to the corresponding range of uniformity of wave surface slope. Since then, it has long been an appraisal that structures are large if $D / L>0.2$ or small if $D / L<0.2$. However, this explanation for the criterion is rather qualitative. Moreover, $D / L<0.15$ was also proposed as the criterion for small structures $[10,11]$.

The foregoing argument implies that the criteria for classification of large and small scale ocean structures are not quantitatively clear. And it can be reasonably inferred that there should be a scale range between large and small scales, which is here termed medium scale. This is one of the task we want to deal with in this paper. Another task is to delineate the dominance of diffraction or viscosity effect for the medium scale structures. Is the wave force of the medium scale structures dominated by viscous or inertial effects or both? Or, is it possible for the two to be ignored?

These issues have been seldom reported. One of the possible reason may be that there has been few marine structures in practice whose dimensions are near $D / L=0.2$. Nonetheless, with the development of ocean engineering, more and more huge structures are necessary. Take the offshore wind power for example. The capacity of offshore wind turbines are becoming larger and larger in order to harvest wind power more efficiently. These turbines appeal to huge supporting structures, monopiles with large diameters or large platforms. This kind of structures are very likely of the dimensions near $D / L=0.2$, belonging to the type of medium scale. Two typical examples of this kind are found in use in China. One is the high-rise pile cap foundation used in Donghai Bridge Windfarm, East China Sea, whose cap is of $14 \mathrm{~m}$ in diameter [12-14]. The other is the composite bucket foundation used in Qidong Offshore Windfarm, Jiangsu Province, China, which is of 25-40 $\mathrm{m}$ in diameter [15]. An additional example is the oyster oscillating wave surge converter (OWSC) as presented by Wei et al. [16], where the ratio of the characteristic length scale of the full scale OWSC to wave length is 0.19 .

Therefore, it is necessary to examine the hydrodynamic classification criterion of offshore structures and delineate correspondingly estimation method for wave loads. For this purpose, the typical fixed and vertical surface-piercing circular cylinder is considered in the present study. So, radiation effect is not involved. Nonetheless, this does not matter the present investigation. On the one hand, the traditional theory of the hydrodynamic classification criterion of offshore structures is based on the analysis of the dominance of diffraction effect on the wave loads on the typical cylinder, excluding radiation effect. On the other hand, even if a floating body is considered where radiation should be involved, the potentials of the incident waves and scattered waves are identical in the fixed body case and, hence, the exciting force is identical to the wave-induced force acting in the fixed body case, as pointed out by Sarpkaya and Isaacson [1].

Therefore, in the present paper, a numerical wave flume is established first, where a vertical surface-piercing circular cylinder is bottom-fixed in the flume. By solving Navier-Strokes $(\mathrm{N}-\mathrm{S})$ equation and Euler equation respectively, the viscous and inertial components of the wave loads on the cylinder are separated accurately. The variation of the viscous and inertial forces with the diameter to wavelength ratio is further studied. Based on these analyses, the medium structure is scaled, and the calculation methods of wave loads for different scales of structures are proposed, with the accuracy delineated quantitatively. 


\section{Numerical wave flume}

\subsection{Governing equation}

A three-dimensional numerical wave flume is established first, in which a circular cylinder is erected vertically and pierces free surface (Fig. 1). The governing equation is the Navier-Stokes equations, which reads,

$$
\left\{\begin{array}{l}
\frac{\partial \rho_{f}}{\partial t}+\nabla \cdot\left(\rho_{f} \boldsymbol{U}\right)=0, \\
\frac{\partial\left(\rho_{f} \boldsymbol{U}\right)}{\partial t}+\nabla \cdot\left(\rho_{f} \boldsymbol{U}\right) \boldsymbol{U}-\nabla \cdot\left(\mu_{f} \nabla \boldsymbol{U}\right)=-\nabla p-\rho_{f} g,
\end{array}\right.
$$

where $\boldsymbol{U}$ is velocity vector, $\rho_{f}$ the density of fluid, $\boldsymbol{g}$ the acceleration vector due to gravitation, $\mu_{f}=\mu+\mu_{t}$ the dynamic viscosity, $\mu$ the physical dynamic viscosity of fluid, $\mu_{t}$ the dynamic viscosity due to turbulence. If $\mu_{f}=0$, Eq. (3) becomes the Euler equation.

The shear stress transfer (SST) $k$ - $\omega$ turbulence model is employed to account for the boundary layer flow near the cylinder so as to model the effect of water viscosity on the wave loads on the cylinder. The governing equations of the model is as follows [17]

$$
\begin{aligned}
\frac{\partial k}{\partial t}+U_{j} \frac{\partial k}{\partial x_{j}}= & P_{k}-\beta^{*} k \omega+\frac{\partial}{\partial x_{j}}\left[\left(v+\sigma_{k} v_{T}\right) \frac{\partial k}{\partial x_{j}}\right], \\
\frac{\partial \omega}{\partial t}+U_{j} \frac{\partial \omega}{\partial x_{j}}= & \alpha S^{2}-\beta \omega^{2}+\frac{\partial}{\partial x_{j}}\left[\left(v+\sigma_{\omega} v_{T}\right) \frac{\partial \omega}{\partial x_{j}}\right] \\
& +2\left(1-F_{1}\right) \sigma_{\omega 2} \frac{1}{\omega} \frac{\partial k}{\partial x_{i}} \frac{\partial \omega}{\partial x_{i}},
\end{aligned}
$$

where $v$ is the viscosity of fluid and $v_{T}$ the eddy viscosity. The constants $\left(\alpha, \beta, \sigma_{k}, \sigma_{\omega}\right)$ in Eqs. (4) and (5), denoted here by $\phi$, are calculated by

$\phi=F_{1} \phi_{1}+\left(1-F_{1}\right) \phi_{2}$,

where $\phi_{1}$ represents the corresponding constant in the original $k-\omega$ model $\left(\alpha_{1}, \beta_{1}, \sigma_{k 1}, \sigma_{\omega 1}\right)$ and $\phi_{2}$ the corresponding constant in the transformed $k-\varepsilon$ model $\left(\alpha_{2}, \beta_{2}, \sigma_{k 2}, \sigma_{\omega 2}\right)$. Additional closure coefficients and relations are as follows

$$
\begin{aligned}
& P_{k}=\min \left(\tau_{i j} \frac{\partial U_{i}}{\partial x_{j}}, 10 \beta^{*} k \omega\right), \\
& F_{1}=\tanh \left\{\left\{\min \left[\max \left(\frac{\sqrt{k}}{\beta^{*} \omega y}, \frac{500 v}{y^{2} \omega}\right), \frac{4 \sigma_{\omega 2} k}{C D_{k \omega} y^{2}}\right]\right\}^{4}\right\}
\end{aligned}
$$

$C D_{k \omega}=\max \left(2 \rho \sigma_{\omega 2} \frac{1}{\omega} \frac{\partial k}{\partial x_{i}} \frac{\partial \omega}{\partial x_{i}}, 10^{-10}\right)$, where $\alpha_{1}=0.553, \alpha_{2}=0.44, \beta_{1}=0.075, \beta_{2}=0.0828$, $\sigma_{k 1}=0.85, \sigma_{k 2}=1.0, \sigma_{\omega 1}=0.5, \sigma_{\omega 2}=0.856, \beta^{*}=0.09$. The eddy viscosity is formulated as

$v_{T}=\frac{a_{1} k}{\max \left(a_{1} \omega, S F_{2}\right)}$,

where $a_{1}=0.31$, and

$S=\sqrt{2 W_{i j} W_{i j}}$,

$W_{i j}=\frac{1}{2}\left(\frac{\partial U_{i}}{\partial x_{j}}-\frac{\partial U_{j}}{\partial x_{i}}\right)$,

$F_{2}=\left[\max \left(\frac{2 \sqrt{k}}{\beta^{*} \omega y}, \frac{500 v}{y^{2} \omega}\right)\right]^{2}$,

in which $y$ is the shortest distance to the no-slip boundary.

The free surface (i.e. the interface between water and air) is modeled through the volume of fluid (VOF) technique as in Ref. [18], which introduces the volume fraction to denote the occupying volume of water or air in the numerical cell, which is defined as

$\alpha= \begin{cases}0, & \text { air, } \\ \in(0,1), & \text { free surface }, \\ 1, & \text { water. }\end{cases}$

Then, the air and water phase calculations are integrated into a unified equation, which reads

$\frac{\partial \alpha}{\partial t}+\alpha \nabla \cdot \boldsymbol{U}+\alpha(1-\alpha) \nabla \cdot \boldsymbol{U}_{r}=0$,

where $\boldsymbol{U}_{r}$ is a relative compression velocity, artificially used to prevent numerical diffusion of the interface. A detailed description of the artificial compression term can be found in Ref. [19]. The motions of air and water are calculated at the same time as a mixed fluid flow, and the local density and dynamic viscosity can be determined as

$\rho_{f}=\alpha \rho+(1-\alpha) \rho_{a}$,

$\mu_{f}=\alpha \mu+(1-\alpha) \mu_{a}$.

where subscript $a$ refers to air.

\subsection{Numerical methods}

The OpenFOAM framework is used for numerical solution of the governing equation. A combined PISO-SIMPLE algorithm proposed by Jasak [20] is adopted to account for the coupling of velocity and pressure. Wave generation and absorption are dealt with by using a relaxation technique [21], which combines fully nonlinear potential wave solver [22] and fully nonlinear wave generation and absorption program 

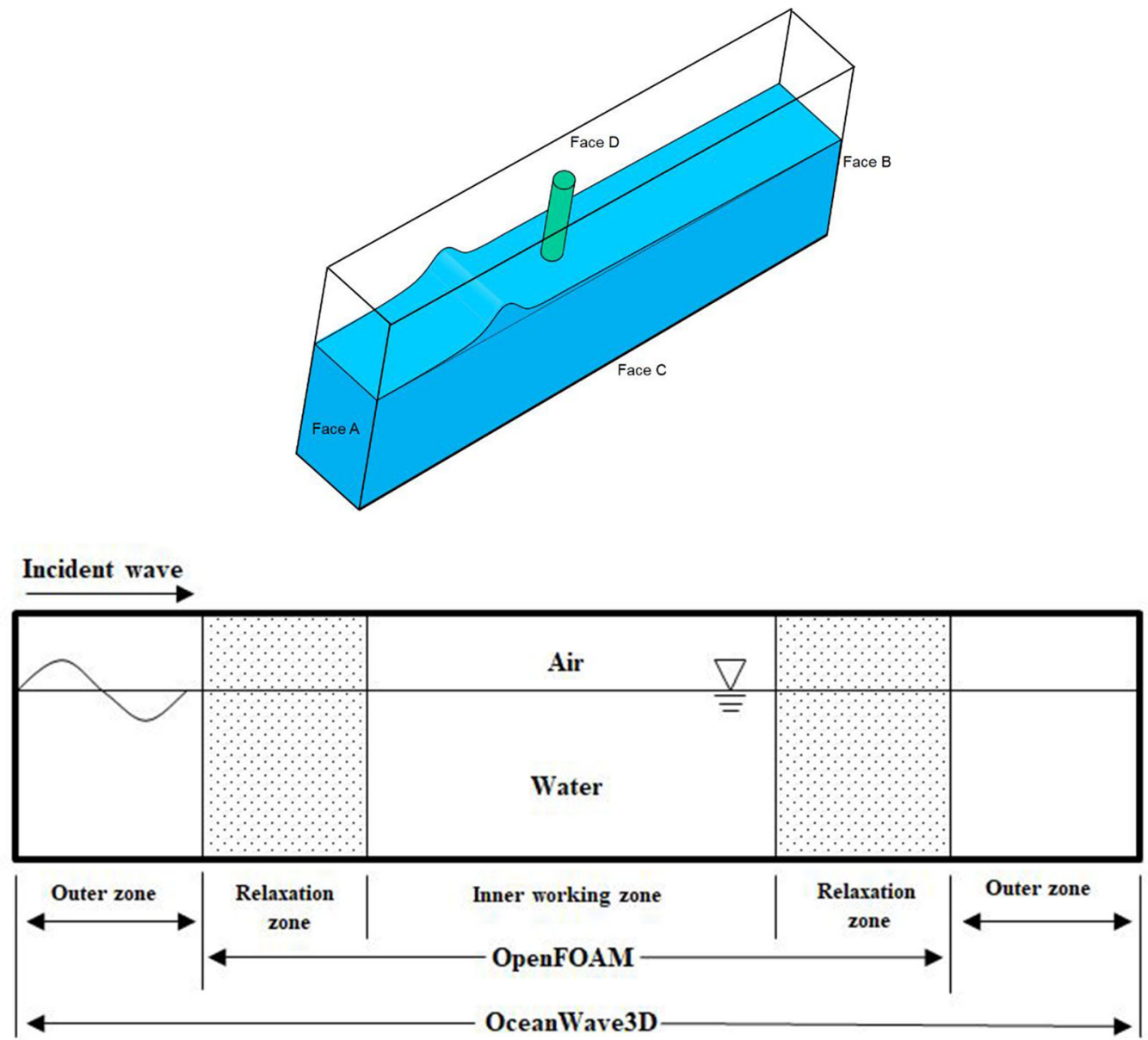

Fig. 1 Sketch of the numerical wave flume

[23]. By this approach, a target wave can be readily generated with a relatively small computational domain and with no worries about wave reflections.

In order to eliminate the reflected and internally generated waves, it is necessary to set relaxation zones at both ends of the flume as shown in Fig. 1. Within the relaxation zone, the velocity $\boldsymbol{U}$ and surface elevation $\eta$ are relaxed in each time step by

$\Phi(x, t)=\xi(\sigma) \Phi_{t}(x, t)+(1-\xi) \Phi_{c}(x, t)$,

where $\Phi$ represents either $\boldsymbol{U}$ or $\eta, \Phi_{t}(x, t)$ and $\Phi_{c}(x, t)$ are the theoretical and computed values of the target wave, respectively, $\xi$ is the relaxation factor defined as

$\xi(\sigma)=1-\frac{\exp \left(\sigma^{3.5}\right)-1}{\exp (1)-1}, \quad \sigma \in[0,1]$

here, $\sigma$ is the normalized horizontal coordinate within the relaxation zones. It equals to zero at the outer ends of the relaxation zones, and unity at the inner ends of the relaxation zones. The relaxation zones should exceed the reflecting wave length so that wave re-reflection is negligible.

\subsection{Boundary and initial conditions}

On the sidewalls, symmetry boundary conditions are specified so as to eliminate the effect of the sidewalls. On the flume bottom, no-slip and no fluid penetration boundary are applied. On the top boundary, the pressure is specified as the atmospheric pressure, and the velocity gradient is zero. On the inlet boundary, the velocity and surface elevation are determined by wave theory with the target wave parameters. On the outlet boundary, no-slip and no fluid penetration boundary are applied (wave absorption is due by the downstream relaxation zone). On the cylinder surface, no-slip and slip boundary conditions are specified for the $\mathrm{N}-\mathrm{S}$ equation and the Euler equation, respectively.

The initial conditions are specified as the calm sea condition. In other words, the free surface is horizontal, the pressure is hydrostatic, and the velocity is zero. 


\section{Computation of wave forces on the cylinder}

Once the solution of the numerical wave flume, in which a surface-piercing circular cylinder is erected, is obtained, the wave force on the cylinder can be readily calculated by integrating the pressure and shear stress over the wetted surface of the cylinder, namely

$\boldsymbol{F}(t)=-\int_{\Omega} p(x, y, z, t) \boldsymbol{n} \mathrm{d} s+\int_{\Omega} \boldsymbol{\tau} \mathrm{d} s$,

where $\boldsymbol{F}(t)$ is the wave force, $p(x, y, z, t)$ is the pressure, $\boldsymbol{n}$ is the outward normal vector of the cylinder surface, $\Omega$ is the wetted area of the cylinder, and $\tau$ is the shear stress on the cylinder surface.

If the solution of the numerical wave flume is obtained based on the N-S equation, Eq. (19) will present the total wave force on the cylinder $\boldsymbol{F}_{N}$. The first term on the righthand side of Eq. (19) accounts for the drag and inertia components. The second term is the friction force, which is negligible compared with the first term. If the solution of the numerical wave flume is obtained based on the Euler equation, the second term on the righthand side of Eq. (19) is zero, and Eq. (19) will present the inertia force $\boldsymbol{F}_{E}$ only. Then, the difference of the two, $\boldsymbol{F}_{N}-\boldsymbol{F}_{E}$, accounts for the effect of viscosity on the wave force. It should be addressed that $\boldsymbol{F}_{N}-\boldsymbol{F}_{E}$ implies the drag force in the following discussions because the friction force is negligible.

\section{Validation of wave generation and wave force estimation}

Validations of the relaxation technique for wave generation and absorption were done by Jacobsen et al. [23] and Paulsen et al. [24]. Additional works are carried out here in order to validate the numerical model and the wave force estimation method.

\subsection{Validation of numerical wave generation}

A wave motion case is designed for validation of wave generation. The target wave is a second-order Stokes wave with a wave height of $0.12 \mathrm{~m}$, a period of $1.5 \mathrm{~s}$ and a wavelength of $2.82 \mathrm{~m}$. The dimension of the numerical wave flume is $10 \mathrm{~m}$ long and $0.65 \mathrm{~m}$ wide. The water depth is $0.5 \mathrm{~m}$. The time history of the numerical free surface elevation at the location of $3 \mathrm{~m}$ away from the wavemaker boundary is compared with the theoretical result as shown in Fig. 2. The agreement is very well, apart from the first period which should be discarded due to the initial conditions of still flume flow.

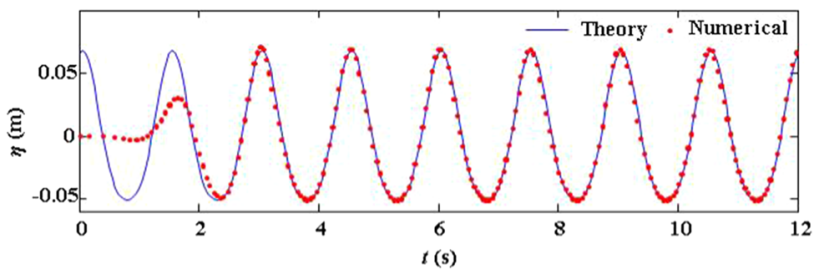

Fig. 2 Comparison between theoretical and numerical free surface elevation at the location of $3 \mathrm{~m}$ away from wavemaker boundary

Table 1 Cases of different grid settings for grid independence examination

\begin{tabular}{llllllr}
\hline Case & A & B & C & D & E & F \\
\hline$P P W L$ & 40 & 50 & 60 & 70 & 70 & 70 \\
$P P W H$ & 15 & 15 & 15 & 15 & 10 & 5 \\
\hline
\end{tabular}
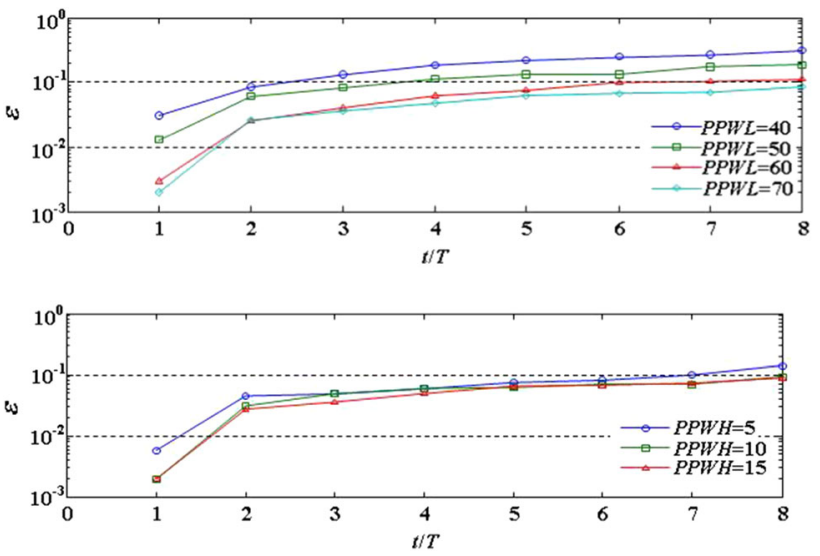

Fig. 3 Relative errors of free surface elevation between theoretical and numerical results of different grids

Grid dependence is examined in order to balance the computational cost and numerical accuracy. The dimension of the numerical wave flume for this purpose is chosen as $30 \mathrm{~m}$ long and $0.65 \mathrm{~m}$ wide. The water depth is $0.5 \mathrm{~m}$. the target wave is the same as in Fig. 2. Grid settings are examined via six cases as listed in Table 1, where $P P W L$ stands for grid number in one wave length and $P P W H$ for grid number in one wave height. A numerical gauge is placed in the inner working zone to monitor wave height history. The relative errors of free surface elevation between theoretical and numerical results of the cases are shown in Fig. 3. It is demonstrated that $P P W L=60$ and $P P W H=10$ are fine enough because finer grids do not improve the accuracy significantly and the attenuation of wave amplitude is less than $10 \%$ after 8 periods.

\subsection{Validation of wave force estimation}

When calculating wave force on a structure of small scale, flow separation and vortex shedding should be carefully dealt 


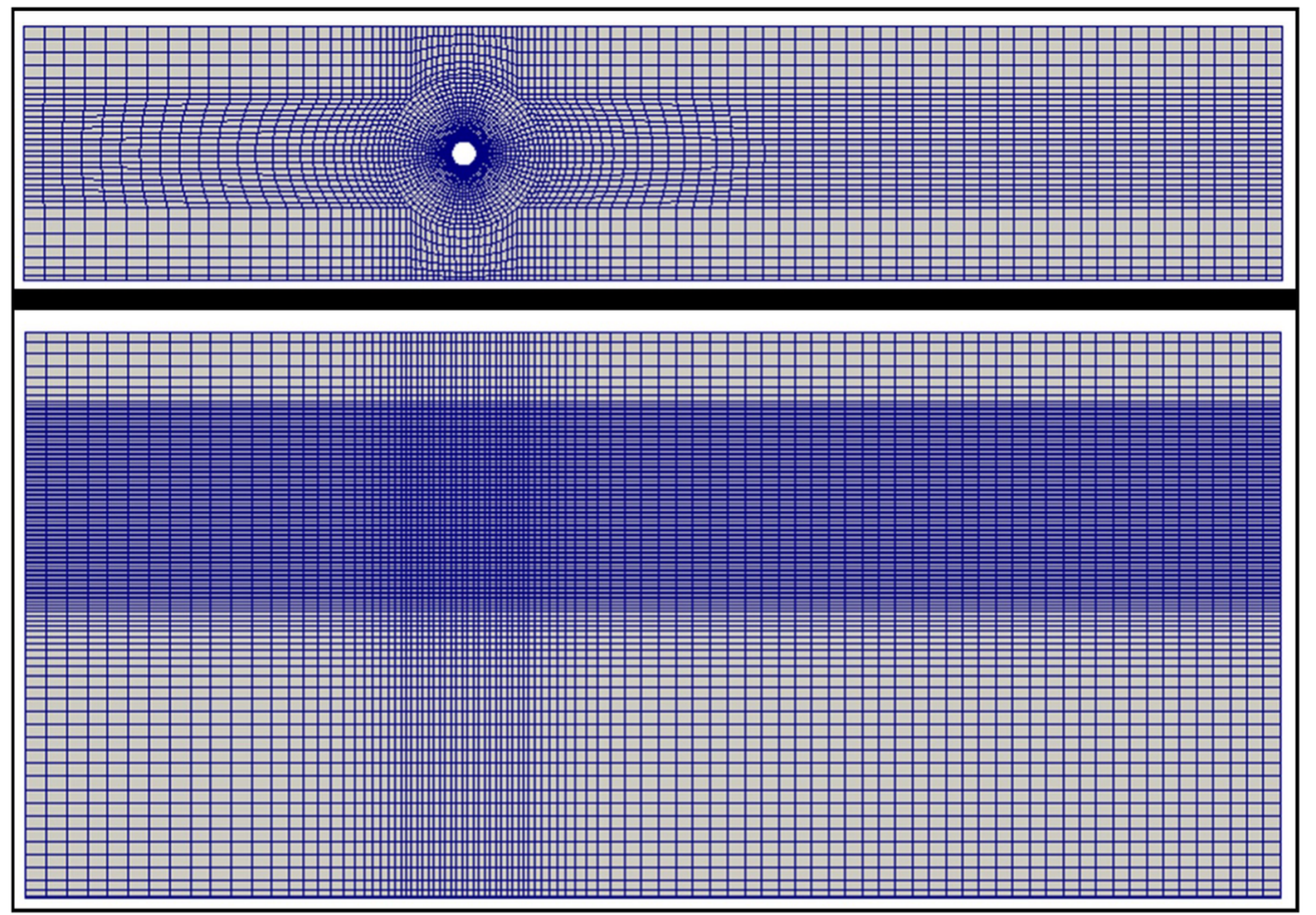

Fig. 4 Mesh near the cylinder (up: top view, down: side view)

with. Therefore, selection of an appropriate turbulence model is very important, which will directly affect the accuracy of the evaluation of drag force. Here, the SST $k-\omega$ turbulence model, which is widely used for wall turbulent flow, is adopted. Meanwhile, grid resolution is also crucial for turbulence modeling. The thickness of the first grid from the wall is determined by

$\Delta=D \Delta^{+} \sqrt{80} R e^{-13 / 14}$,

where $D$ is the diameter of the cylinder, $\Delta^{+} \leq 2-5$ is the nondimensional thickness of the first grid from the wall, $R e$ is the Reynolds number.

Let us first assume a case for validation of wave force estimation for a fixed and vertical surface-piercing cylinder with a diameter of $0.12 \mathrm{~m}$. The numerical wave flume is $8 \mathrm{~m}$ long, $0.8 \mathrm{~m}$ wide and $0.9 \mathrm{~m}$ high. The water depth is $0.6 \mathrm{~m}$. The target wave is a second-order Stokes wave with a wave height of $0.105 \mathrm{~m}$, a period of $1.2 \mathrm{~s}$ and a wavelength of $2.12 \mathrm{~m}$. The mesh near the cylinder is shown in Fig. 4. Finer grids are used near the cylinder and the free surface. Figure 5 shows the numerical and theoretical free surface elevation and the inline force acting on the cylinder. The agreement is seen very well, apart from the first two periods which should be discarded due to the initial conditions of still flume flow.

Validation of turbulence model and near-wall grid resolution are also carried out. The parameters of the validation
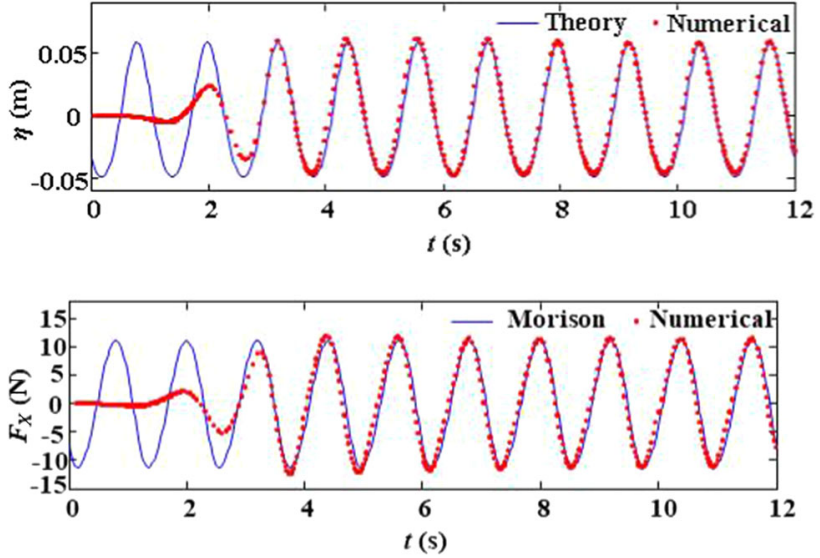

Fig. 5 Comparison between numerical and theoretical results (left: free surface elevation, right: inline force)

case are designed as follows. The diameter of the cylinder is $0.01 \mathrm{~m}$ and the water depth is $0.6 \mathrm{~m}$. The wave is of a wave height of $0.06 \mathrm{~m}$, a period of $1.16 \mathrm{~s}$ and a wavelength of $2 \mathrm{~m}$. After Eq. (20), the thickness of the first grid from the cylinder wall is $\Delta=0.15-0.375 \mathrm{~mm}$. Computations are performed for three cases where $\Delta=0.15 \mathrm{~mm}, \Delta=0.25 \mathrm{~mm}$, $\Delta=0.5 \mathrm{~mm}$, respectively. The inline wave forces of the three cases are plotted in Fig. 6. Grid independence is seen when $\Delta=0.25 \mathrm{~mm}$, which implies $\Delta^{+}=3.3$. Hereafter, $\Delta^{+}<3$ is taken as the criterion for near-wall grid resolution. 


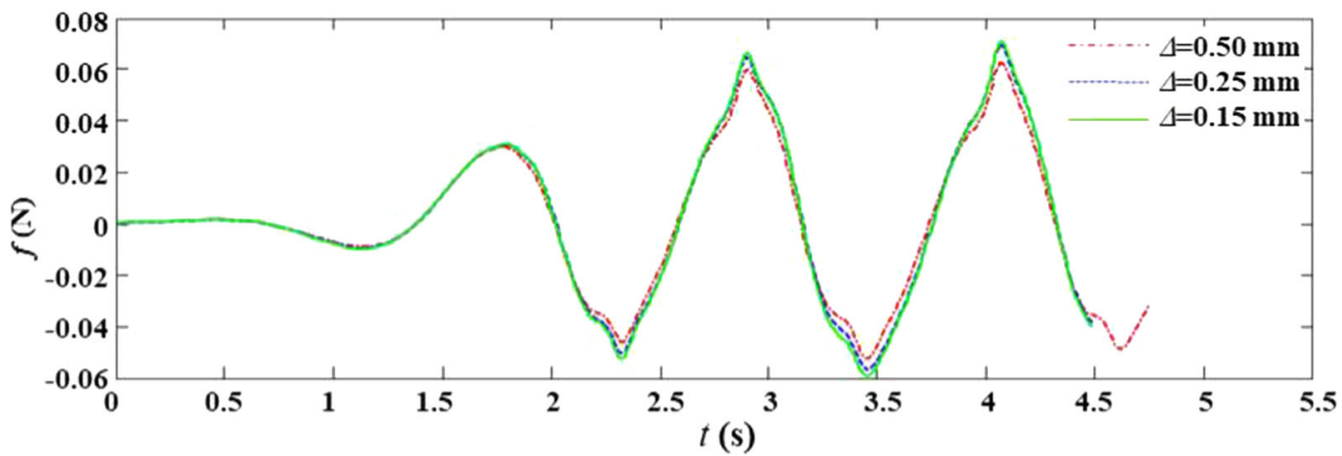

Fig. 6 Influence of near-wall grid thickness on the inline wave force of the cylinder

Table 2 Wave parameters and structure scales

\begin{tabular}{lllllll}
\hline Water depth, $h(\mathrm{~m})$ & Wave height, $H(\mathrm{~m})$ & $\begin{array}{l}\text { Wave number, } k \\
\left(\mathrm{~m}^{-1}\right)\end{array}$ & $k \cdot h$ & $D / L$ & $R e$ & $K c$ \\
\hline 0.60 & 0.06 & 3.133 & 1.88 & 0.0025 & $8.5 \times 10^{2}$ & 37.7 \\
0.60 & 3.133 & 1.88 & 0.005 & $1.7 \times 10^{3}$ & 18.9 \\
0.60 & 3.133 & 1.88 & 0.01 & $3.4 \times 10^{3}$ & 9.42 \\
0.60 & 0.06 & 3.133 & 1.88 & 0.02 & $6.8 \times 10^{3}$ & 4.71 \\
0.60 & 0.06 & 3.133 & 1.88 & 0.05 & $1.7 \times 10^{4}$ & 1.88 \\
0.60 & 0.06 & 3.133 & 1.88 & 0.10 & $3.4 \times 10^{4}$ & 0.94 \\
0.60 & 0.06 & 3.133 & 1.88 & 0.15 & $5.1 \times 10^{4}$ & 0.63 \\
0.60 & 0.06 & 3.133 & 1.88 & 0.20 & $6.8 \times 10^{4}$ & 0.47 \\
0.60 & 0.06 & 3.133 & 1.88 & 0.30 & $1.0 \times 10^{5}$ & 0.31 \\
30.0 & 0.06 & 0.0628 & 1.88 & 0.0025 & $3.0 \times 10^{5}$ & 37.7 \\
30.0 & 3.00 & 0.0628 & 1.88 & 0.005 & $6.0 \times 10^{5}$ & 18.9 \\
30.0 & 3.00 & 0.0628 & 1.88 & 0.01 & $1.2 \times 10^{6}$ & 9.42 \\
30.0 & 3.00 & 0.0628 & 1.88 & 0.02 & $2.4 \times 10^{6}$ & 4.71 \\
30.0 & 3.00 & 0.0628 & 1.88 & 0.05 & $6.0 \times 10^{6}$ & 1.88 \\
30.0 & 3.00 & 0.0628 & 1.88 & 0.1 & $1.2 \times 10^{7}$ & 0.94 \\
30.0 & 3.00 & 0.0628 & 1.88 & 0.15 & $1.8 \times 10^{7}$ & 0.63 \\
30.0 & 3.00 & 0.0628 & 1.88 & 0.2 & $2.4 \times 10^{7}$ & 0.47 \\
30.0 & 3.00 & 0.0628 & 1.88 & 0.3 & $3.6 \times 10^{7}$ & 0.31 \\
\hline & 3.00 & & & & &
\end{tabular}

\section{Dependence of wave force on structure scale}

To delineate variations of drag and inertia components of wave loads on a cylinder, we have simulated 18 cases of different wave parameters and cylinder dimensions, which are listed in Table 2, where $R e=\left(U_{m} D\right) / v, K c=\left(U_{m} T\right) / D$, $K c$ is Keulegan-Carpenter number, $U_{m}$ is the maximum horizontal velocity calculated by linear wave theory. The first 9 cases are used to model laboratory situation, of which the Reynolds number is relatively low, from $8.5 \times 10^{2}$ to $1.0 \times$ $10^{5}$. The other 9 cases are used to model real situation, of which the Reynolds number is relatively high, from $3 \times 10^{5}$ to $3.6 \times 10^{7}$. The water depth and wave parameters of each 9 cases remain unchanged but the cylinder diameters are different. In order to further guarantee avoiding the sidewall effect, the width of flume is set as 10 times the diameter of the cylinder.

\subsection{Effect of cylinder scale on drag force}

Let $f_{N}$ and $f_{E}$ denote the horizontal magnitudes of $\boldsymbol{F}_{N}$ and $\boldsymbol{F}_{E}$, respectively. Then, the weight of the drag force in the total force of the cylinder can be expressed as

$\gamma=\frac{f_{N}-f_{E}}{f_{N}}$.

It is obvious that the weight is a function of the cylinder dimension and time or wave phase. The maximum wave force is of most concern in engineering. The weight at the phase of maximum wave force for different scale of the cylinder is shown in Fig. 7. When $D / L=0.02$, the weight is only $1.83 \%$ 


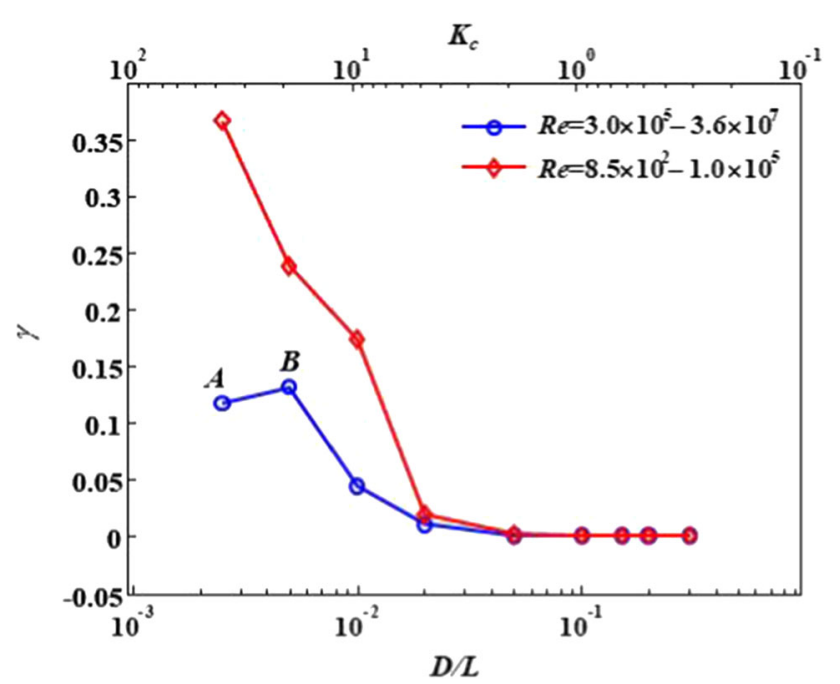

Fig. 7 Weight of the drag force versus $D / L$ and $K c$ number

and $1.01 \%$ for low and high Reynolds number cases, respectively. This implies that the drag force is negligible when $D / L>0.02$ (this is consistent with the result of the full scale OWSC with its flap width to wave length ratio equal to 0.19 , as presented by Wei et al. [16]) and the error of the maximum wave force on the cylinder calculated via potential theory is less than $2 \%$. When $D / L \leq 0.02$, the weight increases rapidly with decreasing $D / L$ and the weight of low Reynolds number is much greater than high Reynolds number, suggesting that the drag force accounts for more and more portion of the total force with decreasing $D / L$ (especially for the case of low Reynolds number). In the case of high Reynolds number, the weight at point $A$ is unexpectedly smaller than at point $B$. This may be explained by flow transition. When the cylinder scale is very small, in a short time interval at the phase of the maximum wave force, the wave-induced flow acts to the cylinder just like steady flow, and the Reynolds number at point $A$ is $3.0 \times 10^{5}$, which is just at the point of resistance crisis in the case of a steady flow over a circular cylinder.

\subsection{Effect of cylinder scale on inertia force}

As already mentioned in the introduction, when a vertical cylinder spans a significant fraction of a wavelength, the incident waves generally undergo significant scattering or diffraction, and the calculations of the wave force should then take such scattering into consideration, especially for the inertia component. Thus, the cylinder scale is an important parameter for the assessment of inertia component of the wave force. The effect of the cylinder scale on the inertia force of the cylinder can be quantified by

$\kappa=\frac{f_{E}-f_{K}}{f_{K}}$,

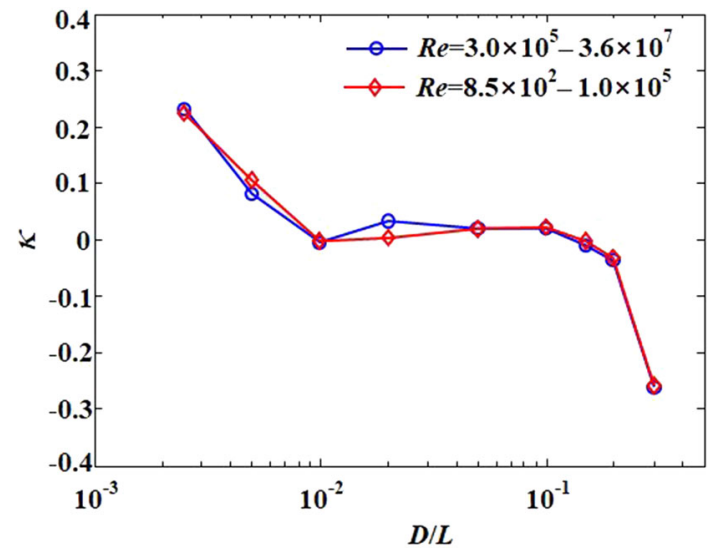

Fig. $8 \kappa$ varies with scale parameter $D / L$

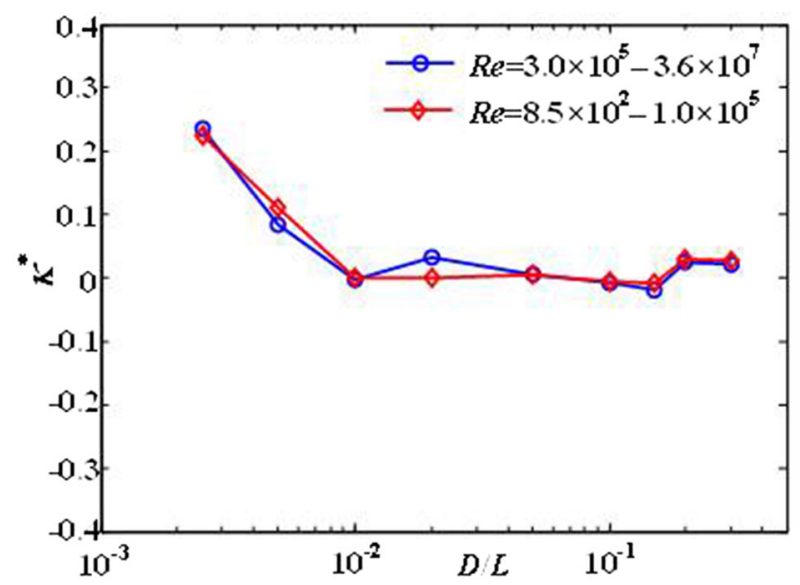

Fig. $9 \kappa *$ varies with scale parameter $D / L$

where $f_{K}$ is the Froude-Krylov force.

The dependence of $\kappa$ on the cylinder scale is shown in Fig. 8, where the wave parameters are listed in Table 2. When $D / L$ is greater than 0.01 and less than $0.15, \kappa$ is approximately zero, suggesting that the disturbance of the cylinder to the wave field is negligible and the inertia force can be calculated by Froude-Krylov approach. When $D / L>0.2, \kappa$ decreases quickly, suggesting that the diffraction should be considered and it makes the inertia force decrease. When $D / L$ is less than $0.01, \kappa$ is positive and increases with decrease of $D / L$, which is unexpected. To explain this unexpected results, we define a new parameter

$\kappa_{*}=\frac{f_{E}-f_{D}}{f_{D}}$,

where $f_{D}$ is the horizontal inertia force calculated with the linear diffraction theory. The dependence of $\kappa_{*}$ on the cylinder scale is shown in Fig. 9. When $D / L$ is greater than $0.01, \kappa_{*}$ is approximately zero which implies that the inertia force is very close to the result calculated by linear diffraction theory, once again validating the results in Fig. 8. However, when 

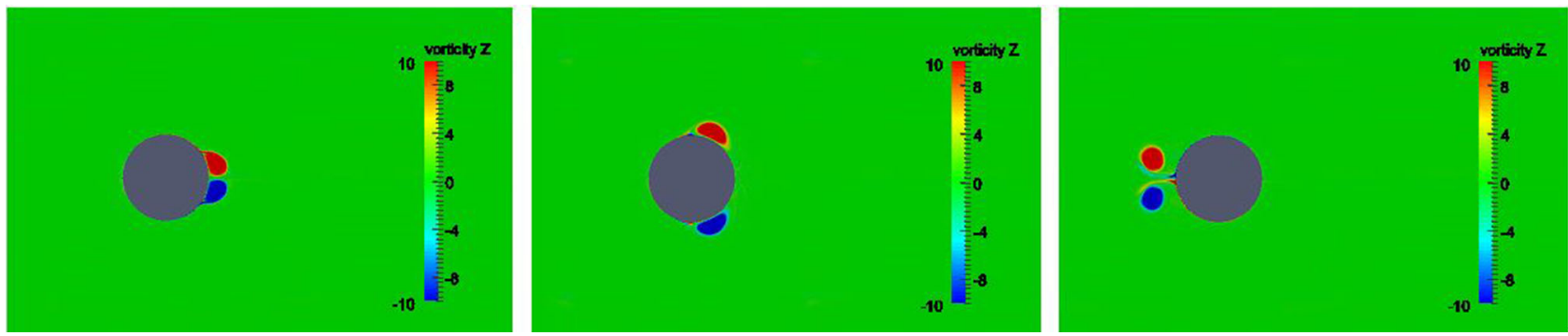

Fig. 10 Vortex contours at three instants in a wave cycle calculated by Euler equation $\left(D / L=0.005, R e=6 \times 10^{5}\right)$

$D / L$ is less than 0.01, the results shown in Figs. 8 and 9 are same to each other. In other words, the unexpected results have nothing to do with diffraction. Further analysis is carried out by extracting vortex data from the numerical results of the Euler equation. Figure 10 demonstrates the vortex contours at three instants in a wave cycle. Vortex shedding can be seen though it is very weak. This is resulted from numerical viscosity, which is inevitable. Nonetheless, numerical viscosity shows its effect only for the cylinder of very small scale.

\section{Classification of offshore structures}

From the above analyses, we can now present a new scaling criterion for offshore structures from hydrodynamics view of point, which classifies the offshore structure into three kinds by carefully quantified limits as follows.

Small-scale structure $D / L \leq 0.02$. The wave load of the structure is dominated by drag force and diffraction is negligible. The wave load can be estimated by the Morison equation.

Medium-scale structure $0.02<D / L<0.2$. Both the viscosity and diffraction effects do not dominate the wave load. The errors of wave load estimation are $2 \%$ and $4 \%$ without considering viscosity and diffraction, respectively. The wave load can be calculated by either diffraction theory or Froude-Krylov equation. The latter approach is of course more convenient and simpler.

Large-scale structure $D / L \geq 0.2$. Diffraction becomes dominant over viscosity effect and the latter is negligible. The wave load of the structure can be calculated based on diffraction theory. It can also be estimated by the Morison equation, but the inertia coefficient should be modified with diffraction theory.

\section{Conclusions}

We have focused in the present paper on the hydrodynamic classification of offshore structures. We have first established a numerical wave flume to model the interaction between waves and a fixed and vertical surface-piercing circular cylinder. Then, we accurately separate the viscous and inertia forces by solving the Navier-Stokes and Euler equations with free surface involved, respectively. Based on detailed quantitative analysis of the dependence of the drag and inertia forces on the structure scale, we put forward a novel concept of medium-scale to renew the criterion for the hydrodynamic classification of offshore structures with meaningful quantitative limits to divide small-, medium- and large-scale structures. If the structure size to wave length ratio is less than 0.02 , the structure is of small-scale and its wave load can be estimated by the Morison equation. If the structure size to wave length ratio is greater than 0.2 , the structure is of large-scale and its wave load can be calculated based on diffraction theory or by the Morison equation with the inertia coefficient modified with diffraction theory. If the structure size to wave length ratio ranges in between 0.02 and 0.2 , the structure is of medium-scale and its wave load can be estimated by the Froude-Krylov equation. By this new rule, one can avoid great difficulties of choosing drag coefficient to estimate wave loads of the medium-scale structures if otherwise using the traditional rule that just classifies offshore structures into small and large structures. The present works are based on linear waves. Further investigation should be made for nonlinear waves.

Acknowledgements This work was supported by the National Key R\&D Program of China (Grant 2017yfc1404200), the National Natural Science Foundation of China (Grant 11572332), and the Strategic Priority Research Program of the Chinese Academy of Sciences (Grants xdb22040203 and xda22000000).

\section{References}

1. Sarpkaya, T., Isaacson, M.: Mechanics of Wave Forces on Offshore Structures. Van Nostrand Reinhold Company (1981)

2. Morison, J.R., Johnson, J.W., Schaaf, S.A.: The force exerted by surface waves on piles. J. Petrol. Technol. 2(05), 149-154 (1950)

3. Kang, L., Ge, F., Wu, X., et al.: Effects of tension on vortex-induced vibration (VIV) responses of a long tensioned cylinder in uniform flows. Acta. Mech. Sin. 033(001), 1-9 (2017) 
4. Lyu, G., Zhang, H., Li, J.: Effects of incident wind/wave directions on dynamic response of a spar-type floating offshore wind turbine system. Acta. Mech. Sin. 35(5), 954-963 (2019)

5. Fernandes, A.C., Asgari, P., Sales Junior, J.S.: Linear and nonlinear roll damping of an FPSO via system identification of a third order equation with sway-roll coupled damping effects. Ocean Eng. 166, 191-207 (2018)

6. Ma, Z., Wang, S., Wang, Y., et al.: Experimental and numerical study on the multi-body coupling dynamic response of a novel serbuoys-tlp wind turbine. Ocean Eng. 192, 106570-106570 (2019)

7. MacCamy, R.C., Fuchs, R.A.: Wave Forces on Piles: a Diffraction Theory. Corps of Engineers Washington DC Beach Erosion Board (1954)

8. Hogben, N.: Wave loads on structures. In: Proc. Conf. Behavior of Off-Shore Structures, BOSS'76, Trondheim, 1, 187-219 (1976)

9. Chakrabarti. S.K., Tam, W.A.: Cross and local wave loads on a large vertical cylinder-theory and experiment. In: Proc. 7th Offshore Technol, Houston, 1818 (1973)

10. Chakrabarti, S.: Handbook of Offshore Engineering: vol. I. Elsevier, Kidlington (2005)

11. Li, Y.C., Teng, B.: Wave Action on Maritime Structures. China Ocean Press (2012)

12. Zhou, J.F., Lin, Y.F.: Essential mechanics issues of offshore wind power systems. Sci Sin-Phys Mech Astron. 43, 1589-1601 (2013). https://doi.org/10.1360/132013-335. (in Chinese)

13. Chen, L., Zhou, J.F.: Nonlinear wave loads on high-rise pile cap structures in the Donghai Bridge Wind Farm. Int. J. Offshore Polar Eng. 28(3), 263-271 (2018). https://doi.org/10.17736/ijope.2018. jc717

14. Zhou, J.F., Wang, X.: Effects of cap bottom elevation on wave loads on the piles under the cap. In: Proceedings of the ASME 2019 38th International Conference on Ocean, Offshore and Arctic Engineering, Glasgow, Scotland, June 9-14 (2019)
15. Lian, J.J., Chen, F., Yang, X., et al.: Suction installation and leveling of composite bucket foundation for offshore wind turbines. Journal of Tianjin University (Science and Technology) 47(11), 987-993 (2014). https://doi.org/10.11784/tdxbz201306050. (in Chinese)

16. Wei, Y.J., Rafiee, A., Henry, A., et al.: Wave interaction with an oscillating wave surge converter. Part I: Viscous effects. Ocean Engineering 104, 185-203 (2015)

17. Menter, F.R.: Two-equation eddy-viscosity turbulence models for engineering applications. AIAA Journal 32, 1598-1605 (1994)

18. Hirt, C.W., Nichols, B.D.: Volume of fluid (VOF) method for the dynamics of free boundaries. J. Comput. Phys. 39, 201-225 (1981)

19. Weller, H.G., Tabor, G., Jasak, H., et al.: A tensorial approach to computational continuum mechanics using object-oriented techniques. Computers in Physics 12(6), 620-631 (1988)

20. Jasak, H.: Error analysis and estimation for the Finite Volume Method with applications to fluid flows. [Ph.D. Thesis] University of London (1996)

21. Paulsen, B.T., Bredmose, H., Bingham, H.B.: An efficient domain decomposition strategy for wave loads on surface piercing circular cylinders. Coast. Eng. 86, 57-76 (2014)

22. Engsig-Karup, A.P., Bingham, H.B., Lindberg, O.: An efficient flexible-order model for 3D nonlinear water waves. J. Comput. Phys. 228, 2100-2118 (2009)

23. Jacobsen, N.G., Fuhrman, D.R., Freds $\varnothing$, J.: A wave generation toolbox for the opeN-Source CFD library: openfoam ${ }^{\circledR}$. Intl J. Numer. Meth. Fluids 70(9), 1073-1088 (2012)

24. Paulsen, B.T., Bredmose, H., Bingham, H.B., et al.: Forcing of a bottom-mounted circular cylinder by steep regular water waves at finite depth. J. Fluid Mech. 755, 1-34 (2014) 